Discrete Comput Geom 34:269-284 (2005)

DOI: $10.1007 / \mathrm{s} 00454-005-1171-5$

\title{
Real Root Conjecture Fails for Five- and Higher-Dimensional Spheres*
}

\author{
Światosław R. Gal \\ Department of Mathematics, Wrocław University, \\ pl. Grunwaldzki 2/4, 50-384 Wroclaw, Poland \\ sgal@math.uni.wroc.pl
}

Communicated by Louis J. Billera

\begin{abstract}
A construction of convex flag triangulations of five- and higher-dimensional spheres, whose $h$-polynomials fail to have only real roots, is given. We show that there is no such example in dimensions lower than five. A condition weaker than real rootedness is conjectured and some evidence is provided.
\end{abstract}

\section{Introduction}

Let the $f$-polynomial $f_{X}$ of a simplicial complex $X$ be defined by the formula

$$
f_{X}(t):=\sum_{\sigma \in X} t^{\# \sigma}
$$

There is a classical problem: what can be said in general about the f-polynomials of (a certain class of) simplicial complexes?

In particular, it is well known what polynomials appear as $f$-polynomials of

- general simplicial complexes or

- triangulations of spheres that are the boundary complexes of convex polytopes

(the reader may consult [Sta1] for ample discussion). The question concerning all triangulations of spheres still remains open. However, the answer is conjecturally the same.

* This research was supported by KBN Grant 2 P03A 01725. 
What we are interested in is the special case of the latter. Namely, what can be said in general about the $f$-polynomials of flag sphere triangulations?

The paper is organized as follows. In Section 1.1 we recall the definition of and basic facts about flag complexes. In Section 1.2 we discuss the generalized homology spheres (GHS), also called Gorenstein* complexes, a suitable generalization of sphere triangulations. In Section 2.1 we define a substitution in the $f$-polynomial of a GHS which we call the $\gamma$-polynomial and find very useful when the complex is flag. In Sections 2.2 and 2.3 we discuss the relation of a $\gamma$-polynomial to the well-known Charney-Davis Conjecture and the cd-index. In Section 2.4 we explore the construction of edge subdivision which will be used in later proofs. In Section 3.1 we formulate the Real Root Conjecture (a strengthening of the well-known Charney-Davis Conjecture) and prove it for generalized homology spheres of dimension at most four. In Section 3.2 we prove a partial converse. This provides an almost complete answer to the question posed in the previous paragraph in low dimensions. In Section 3.4 we construct a flag convex triangulation of $S^{5}$ that is a counterexample to the Real Root Conjecture.

Apart from understanding $f$-polynomials of flag triangulation of spheres the Real Root Conjecture was important since the affirmative answer, as observed by Reiner and Welker [RW], would provide a partial check for the Neggers-Stanley Conjecture.

We conjecture that the coefficients of the $\gamma$-polynomial of the flag GHS are nonnegative. We show that this conjecture is stronger than the Charney-Davis Conjecture and weaker than the Real Root Conjecture, thus it could remain true in all dimensions. One should think of the conjecture on a $\gamma$-polynomial as a lower bound conjecture for flag sphere triangulations. Some evidence is provided.

\section{Preliminaries}

\subsection{Flag Complexes}

Definition 1.1.1. A simplicial complex $X$ with the vertex set $S$ is called flag if for any $T \subset S$ such that $T$ is a clique (i.e., any two distinct vertices of $T$ are joined by an edge), $T$ is a face of $X$.

Obviously a flag complex is determined by its one-skeleton. A barycentric subdivision of any polytopial complex or, more generally, regular CW-complex is flag.

A motivation for studying flag complexes is a theorem of Gromov, which states that a cubical complex is locally CAT(0) if and only if the link of any vertex is flag [Gr]. Recall that locally $\mathrm{CAT}(0)$ space is in particular aspherical.

Definition 1.1.2. The link $L k_{\sigma}$ of a simplex $\sigma$ in a simplicial complex $X$ consists of all $\tau \in X$ such that $\sigma \cup \tau \in X$ and $\sigma \cap \tau=\emptyset$.

Remark 1.1.3. The link of any simplex in a flag complex is flag itself. 


\subsection{The h-Polynomial}

Definition 1.2.1. A (simplicial) generalized homology sphere of dimension $n$ (GHS ${ }^{n}$ ) is a simplicial complex such that the link of any simplex $\sigma$ has the homology of a sphere of dimension $(n-\# \sigma)$. We omit the superscript if not necessary.

A simplicial GHS is also called a Gorenstein* complex (see Theorem 5.2 in Chapter II of [Sta1] for further reference).

Remark 1.2.2. A triangulation of a (homology) sphere is a generalized homology sphere.

We introduce Definition 1.2.1, since the double suspension of a GHS is a triangulation of a sphere [C]. Thus any GHS may appear as the link of an edge in some triangulation of a sphere. More generally, assume that the dimension of a simplicial (or cubical) complex is greater than two. Then it is a cellulation of a manifold if and only if the link of any vertex is a simply connected GHS [E], [F].

Definition 1.2.3. We say that $X$ is a convex sphere triangulation if it is the boundary complex of some convex polytope.

Remark 1.2.4. The link of any simplex of a convex sphere triangulation is also a convex sphere triangulation.

Theorem (Dehn-Sommerville Relations). If $X$ is a $\mathrm{GHS}^{n-1}$ then $f_{X}(t-1)=$ $(-1)^{n} f_{X}(-t)$.

Proof [Kl]. In fact, one needs a weaker assumption. Namely, that that $X$ is Eulerian, i.e. the Euler characteristic of the link of any simplex $\sigma$ equals that of the sphere of an appropriate dimension, or in other words

$$
\sum_{\tau \supset \sigma}(-1)^{\# \tau}=(-1)^{n}
$$

If $\tau$ is a simplex then $(1+s)^{\# \tau}=\sum_{\sigma \subset \tau} s^{\# \sigma}$. Therefore

$f_{X}(t-1)=\sum_{\tau}(t-1)^{\# \tau}=\sum_{\sigma \subset \tau}(-t)^{\# \sigma}(-1)^{\# \tau}=\sum_{\sigma}(-t)^{\# \sigma}(-1)^{n}=(-1)^{n} f_{X}(-t)$.

If $X$ is a $\mathrm{GHS}^{n-1}$, then a more efficient invariant is the $h$-polynomial defined as

$$
(1+t)^{n} h_{X}\left(\frac{1}{1+t}\right):=t^{n} f_{X}\left(\frac{1}{t}\right) .
$$

The Dehn-Sommerville relations written in terms of the $h$-polynomial say that $h_{X}$ is reciprocal (i.e. $h_{X}(t)=t^{n} h_{X}(1 / t)$ ). Moreover, if $X$ is a convex sphere triangulation then $h_{X}$ has a geometric interpretation in terms of a generic height function and $h_{X}\left(t^{2}\right)$ is the Poincare series of the cohomology of the corresponding toric variety [Sta1]. 
Remark 1.2.5. The $h$-polynomial $h_{X}$ is usually defined for any simplicial complex. Although in this exposition we use it only when $X$ is a GHS, the reader should be aware that some formulas (e.g. the formula for the $h$-polynomial of the subdivision along an edge) are incorrect when applied to the general complex.

\section{The $\gamma$-Polynomial}

\subsection{The Definition}

Proposition 2.1.1. Assume that $h$ is a reciprocal polynomial of degree $n$. Then there exists a unique polynomial $\gamma$ of degree at most $\lfloor n / 2\rfloor$ with the property

$$
h(t)=(1+t)^{n} \gamma\left(\frac{t}{(1+t)^{2}}\right) .
$$

Moreover if $h$ has integral coefficients then so does $\gamma$.

Proof. Observe that the dimension of the space of reciprocal polynomials of degree $n$ is $\lfloor n / 2\rfloor+1$. The polynomials $t^{i}(1+t)^{n-2 i}$ for $0 \leq i \leq n / 2$ are reciprocal and linearly independent (being of different degree). Thus they constitute a basis of this space. Since the leading coefficients are equal to one, they also constitute a basis over the integers. The coefficients of $\gamma$ are the coefficients of $h$ with respect to this basis.

Remark 2.1.3. If $h$ is monic then the constant coefficient of $\gamma$ equals one.

Definition 2.1.4. $\quad$ Let $X$ be a GHS (or at least Eulerian). The polynomial $\gamma_{X}$ defined by

$$
h_{X}(t)=(1+t)^{\operatorname{deg} h_{X}} \gamma_{X}\left(\frac{t}{(1+t)^{2}}\right)
$$

is called the $\gamma$-polynomial of $X$.

Question 2.1.6. What is the combinatorial/geometric interpretation of (the coefficients of) the $\gamma$-polynomial?

Conjecture 2.1.7. If $X$ is a flag GHS then all the coefficients of the $\gamma$-polynomial $\gamma_{X}$ are nonnegative.

In the rest of this section we provide some evidence for Conjecture 2.1.7.

Definition 2.1.8. The join of two complexes $X$ and $Y$ on disjoint ground sets is defined as the complex $\{\sigma \cup \tau \mid \sigma \in X, \tau \in Y\}$.

Remark 2.1.9. Any of $f_{\bullet}, h_{\bullet}$ and $\gamma_{\bullet}$ is multiplicative with respect to the joins. 
Corollary 2.1.10. If $X$ and $Y$ satisfy Conjecture 2.1.7 then so does their join $X * Y$.

The Generalized Lower Bound Conjecture [Sta1, Conjecture 6.2 in Chapter II]. Let $X$ be a GHS. Then $h_{X}$ is unimodal, i.e. if $h_{X}(t)=\sum_{i=0}^{n} h_{i} t^{i}$ then $h_{\lfloor n / 2\rfloor} \geq \cdots \geq h_{2} \geq$ $h_{1} \geq h_{0}=1$.

Remark 2.1.11. The above conjecture is true if $X$ is a convex triangulation of a sphere [Sta1].

Corollary 2.1.12. If Conjecture 2.1.7 holds for $X$ then $h_{X}$ is unimodal.

Proof. Each of the polynomials $t^{i}(1+t)^{n-2 i}$ is unimodal. A sum of reciprocal unimodal polynomials is unimodal.

Definition 2.1.13. The cross polytope $O^{n}$ is the $n$-fold join of the zero-dimensional sphere.

Lemma 2.1.14. Let $X$ be a flag $\mathrm{GHS}^{n-1}$ and let $\gamma_{X}(t)=\sum_{i=0}^{n} \gamma_{i} t^{i}$. Then

(1) $\gamma_{1} \geq 0$,

(2) if $\gamma_{1}=0$ then $X$ is a cross-polytope.

Proof. Part (1) is equivalent to the condition that $X$ has at least $2 n$ vertices. We prove this by induction on the dimension of $X$. Take two vertices $v$ and $w$ not joined by an edge. The vertices adjacent to $v$ are vertices of the link of $v$, and, by induction, there are at least $2(n-1)$ of them. Together with $v$ and $w$ there are $2 n$ of them, as desired. This also proves (2).

\subsection{Charney-Davis Conjecture}

Conjecture 2.1.7 is a strengthening of the following well known conjecture.

Charney-Davis Conjecture [CD]. If $X$ is a flag $\operatorname{GHS}^{2 n-1}$ then $(-1)^{n} h_{X}(-1) \geq 0$.

Precisely, $(-1)^{n} h_{X}(-1)$ equals the highest coefficient of $\gamma_{X}$. Indeed, (2.1.5) can be rewritten as

$$
\left(\frac{1}{t}\right)^{n} h_{X}(t)=\left(\frac{(1+t)^{2}}{t}\right)^{n} \gamma_{X}\left(\frac{t}{(1+t)^{2}}\right)
$$

Passing to the limit $t \rightarrow-1$ we obtain the claim.

The Charney-Davis Conjecture, obvious for $n=1$, is proven for $n=2$ [DO]. It is motivated by being a consequence of the Euler Characteristic Conjecture which says that the sign of the Euler characteristic of a $2 n$-dimensional closed aspherical manifold is $(-1)^{n}$. 
Lemma 2.2.1. The sum of the f-polynomials of the links of all simplices with $k$ vertices of any simplicial complex $X$ is equal to $f_{X}^{(k)} / k$ !.

Proof. For any $\tau \in \mathrm{Lk}_{\sigma}$ define $\tau^{*}=\tau \cup \sigma$. We have

$$
\sum_{\substack{\sigma \in X \\
\# \sigma=k}} f_{\mathrm{Lk}_{\sigma}}(t)=\sum_{\substack{\sigma \subset \tau^{*} \in X \\
\# \sigma=k}} t^{\# \tau^{*}-k}=\sum_{\tau^{*} \in X}\left(\begin{array}{c}
\# \tau^{*} \\
k
\end{array}\right) t^{\# \tau^{*}-k}=\frac{f_{X}^{(k)}(t)}{k !} .
$$

Corollary 2.2.2. If $X$ is an even-dimensional GHS then the highest coefficient of $\gamma_{X}$ is nonnegative provided the Charney-Davis Conjecture is true for links of vertices of $X$.

Proof. Let $\operatorname{dim} X=2 n$. By definition

$$
\sum_{i=0}^{n} \gamma_{i} t^{i}(t+1)^{2 n-2 i+1}=(t-1)^{2 n+1} f_{X}\left(\frac{1}{t-1}\right) .
$$

Differentiate and substitute $t=-1$. Since $f_{X}\left(-\frac{1}{2}\right)=0$ (by the Dehn-Sommerville relations) one observes that

$$
\gamma_{n}=(-1)^{n}\left(\frac{1}{2}\right)^{2 n-1} f_{X}^{\prime}\left(-\frac{1}{2}\right) \geq 0,
$$

where the last inequality follows from the fact that $2^{2 n} f_{Y}\left(-\frac{1}{2}\right)=h_{Y}(-1)$ for any $(2 n-1$ dimensional) link $Y$ of any vertex in $X$.

Corollary 2.2.3. If $X$ is a flag GHS of dimension less than five then Conjecture 2.1.7 holds.

Proof. Let $(n-1)$ be the dimension of the GHS. Observe that if $n \leq 5$ then $\gamma_{X}$ is at most quadratic. Write $\gamma_{X}(t)=1+\gamma_{1} t+\gamma_{2} t^{2}$. The linear term $\gamma_{1}$ is nonnegative by Lemma 2.1.14(1). Thus the claim follows for $n \leq 3$. If $n=4$ then $\gamma_{2}$ is nonnegative by the Davis-Okun Theorem. If $n=5$ then $\gamma_{2}$ is nonnegative by the Davis-Okun Theorem and Corollary 2.2.2.

\subsection{The cd-Index}

The reader may find the combinatorial background of this section in Chapter III.4 of [Sta1] and in [Sta2].

Let $P$ be a finite graded poset of rank $n+1$ with $\hat{0}$ and $\hat{1}$. Let $\rho$ be the rank function. For any chain $C$ of the form $\hat{0}<x_{1}<\cdots<x_{d}<\hat{1}$ define a noncommutative monomial $u_{C}=\prod_{i=1}^{n} u_{i}$ in the variables $a$ and $b$ putting

$$
u_{i}=\left\{\begin{array}{lll}
a & \text { if } \quad i \neq \rho\left(x_{k}\right) & \text { for any } \mathrm{k} \\
b & \text { if } \quad i=\rho\left(x_{k}\right) & \text { for some } \mathrm{k}
\end{array}\right.
$$

Finally let

$$
\Upsilon_{P}(a, b):=\sum_{C} u_{C}
$$


and

$$
\Psi_{P}(a, b):=\Upsilon_{P}(a-b, b) .
$$

One may think that $\Upsilon_{P}$ is a generalization of an $f$-polynomial, while $\Psi_{P}$ is that of an $h$-polynomial in the following sense. Define the nerve or the order complex $N(P)$ of $P$ to be the simplicial complex with the set of vertices $P-\{\hat{0}, \hat{1}\}$ such that $C \subset P-\{\hat{0}, \hat{1}\}$ is in $N(P)$ if and only if $C$ is a chain. Then

$$
\begin{aligned}
& f_{N(P)}(t)=\Upsilon_{P}(1, t), \\
& h_{N(P)}(t)=\Psi_{P}(1, t) .
\end{aligned}
$$

Note that $N(P)$ is always a flag complex.

Definition 2.3.1. A graded poset $P$ as above is called Eulerian if for any $x<z$ one has

$$
\sum_{x \leq y \leq z}(-1)^{\rho(y)}=0
$$

Proposition [Sta1], [Sta2] (Bayer-Billera, Fine, Stanley). If $P$ is an Eulerian poset then $\Psi_{P}(a, b)$ may be written as $\Phi_{P}(c, d)$ in $c=a+b$ and $d=a b+b a$.

The noncommutative polynomial $\Phi_{P}$ is called the $c d$-index of $P$. It follows directly from the definition that

$$
\gamma_{N(P)}(t)=\Phi_{P}(1,2 t)
$$

Remark 2.3.2. Babson was the first to notice that $h_{N(P)}(-1)=\Psi_{P}(0,-2)$, i.e. if $n=2 m$ then $(-1)^{m} h_{N(P)}(-1)$ equals $2^{m}$ times the coefficient of $d^{m}$ in $\Psi_{P}$.

The main conjecture on the cd-index is the following:

Conjecture 2.3.3 [Sta2, Conjecture 2.1]. The coefficients of the cd-index of any Gorenstein* poset are nonnegative.

As proven by Stanley [Sta2], if $P$ is a face poset of an $S$-shellable cell (e.g. a convex cell), then Conjecture 2.3.3 holds for $P$. In this case $N(P)$ is the barycentric subdivision of that cell.

Corollary 2.3.4. If $X$ is the barycentric subdivision of an S-shellable cell then Conjecture 2.1.7 holds.

Recently Karu [Kar] has announced the proof of Conjecture 2.3.4. As a corollary we obtain:

Corollary 2.3.5. If $X$ is the barycentric subdivision of a regular CW-sphere then Conjecture 2.1.7 holds. 


\subsection{Edge Subdivision}

Definition 2.4.1. Let $X$ be a simplicial complex. Let $\eta=\{s, t\}$ be an edge. Define $\operatorname{Sub}_{\eta}(X)$ to be a simplicial complex constructed from $X$ by bisection of all simplices containing $\eta$. In other words, let $e$ be any letter not in the vertex set $S$ of $X$. Then $S \cup\{e\}$ is the vertex set of $\operatorname{Sub}_{\eta}(X)$ and

$$
\operatorname{Sub}_{\eta}(X)=\{\sigma \mid \eta \not \subset \sigma \in X\} \cup\left\{\sigma \cup\{e\}, \sigma \cup\{s, e\}, \sigma \cup\{t, e\} \mid \sigma \in \mathrm{Lk}_{\eta}\right\} .
$$

We say that $\operatorname{Sub}_{\eta}(X)$ is the subdivision of $X$ along $\eta$.

The geometric realizations of $X$ and $\operatorname{Sub}_{\eta}(X)$ are homeomorphic. In particular, if $X$ is a sphere triangulation then so is $\operatorname{Sub}_{\eta}(X)$.

Proposition 2.4.2. Assume that $X$ is a convex sphere triangulation. Then $\operatorname{Sub}_{\eta}(X)$ may be realized as a convex sphere triangulation.

Proof. Take any vector $v$ starting at the midpoint of $\eta$ and pointing inside $X$. Taking $e$ to be a sufficiently small translation of the midpoint of $\eta$ in the direction of $-v$ we obtain that the boundary of the convex hull of the vertices of $X$ and $e$ is a (convex) realization of $\operatorname{Sub}_{\eta}(X)$.

Proposition 2.4.3. Subdividing a GHS X along an edge $\eta$ affects $h$ and $\gamma$ as follows:

$$
\begin{aligned}
& h_{\mathrm{Sub}_{\eta}(X)}(t)=h_{X}(t)+t h_{\mathrm{Lk}_{\eta}}(t), \\
& \gamma_{\mathrm{Sub}_{\eta}(X)}(t)=\gamma_{X}(t)+t \gamma_{\mathrm{Lk}_{\eta}}(t) .
\end{aligned}
$$

Proof. Clearly $h_{\operatorname{Sub}_{\eta}(X)}-h_{X}$ does not depend on $X$ but only on the link of $\eta$. Therefore it suffices to check (2.4.4) in one particular case. Define $X$ to be the join of $Y$ and the $k$-gon. Let $\eta$ be an edge of the $k$-gon. Then $\operatorname{Lk}_{\eta}=Y$ and $\operatorname{Sub}_{\eta}(X)$ is the join of $Y$ and the $(k+1)$-gon. Thus the claim follows from Remark 2.1.9 and simple calculation that shows that if $\Delta_{m}$ is an $m$-gon, then

$$
\begin{aligned}
& f_{\Delta_{m}}(t)=1+m\left(t+t^{2}\right), \\
& h_{\Delta_{m}}(t)=1+(m-2) t+t^{2}, \\
& \gamma_{\Delta_{m}}(t)=1+(m-4) t .
\end{aligned}
$$

Proposition 2.4.6. If $X$ is flag then so is $\operatorname{Sub}_{\eta}(X)$.

Proof. Let $\sigma$ be a clique in $\operatorname{Sub}_{\eta}(X)$. If $e \notin \sigma$, then $\sigma$ is a clique in $X$. Thus $\sigma \in X$, but then, by construction, $\sigma \in \operatorname{Sub}_{\eta}(X)$.

Now assume that $e \in \sigma$. Define $\sigma_{*}:=\sigma-\{e, s, t\}$. Each of the vertices in $\sigma_{*}$ being connected by an edge to $e$ has to be connected to $s$ and $t$. Thus $\sigma_{*}$ is a clique in $\mathrm{Lk}_{\eta}$, and, in particular, a simplex in $X$.

On the other hand, $\eta \not \subset \sigma$, since, by definition, $\eta$ is not an edge of $\operatorname{Sub}_{\eta}(X)$. Thus $\sigma$ is of the form $\sigma_{*} \cup\{t, e\}, \sigma_{*} \cup\{e\}$ or $\sigma_{*} \cup\{e, s\}$. Therefore $\sigma \in \operatorname{Sub}_{\eta}(X)$.

Corollary 2.4.7. If $X$ and $\mathrm{Lk}_{\eta}$ satisfy Conjecture 2.1.7, then so does $\operatorname{Sub}_{\eta}(X)$. 


\section{Real Roots}

\subsection{The Conjecture}

Stating Conjecture 2.1.7 we were motivated by

The Real Root Conjecture. The zeros of the h-polynomial of a flag GHS are all real.

Remark 3.1.1. The Real Root Conjecture implies Conjecture 2.1 .7 by the following argument. Assume that a reciprocal polynomial $h$ and a polynomial $\gamma$ are related by (2.1.2). Then $h$ has only real negative roots if and only if the same holds for $\gamma$ because $t /(1+t)^{2}$ is real negative or infinite if and only if $t$ is real negative. The "if" part is obvious. Conversely, if $t /(1+t)^{2}=1 /\left(\sqrt{t}+(\sqrt{t})^{-1}\right)^{2}$ is real negative then $\sqrt{t}+(\sqrt{t})^{-1}$ is purely imaginary. Since $\operatorname{Re}(z)$ and $\operatorname{Re}\left(z^{-1}\right)=\operatorname{Re}(z) /|z|^{2}$ have the same sign, $\sqrt{t}$ has to be purely imaginary. Thus $t$ is real negative.

The Real Root Conjecture was stated by Januszkiewicz in a series of questions concerning $L^{2}$-cohomology of buildings (the details may be found in [DDJO]).

Independently, Reiner and Welker observed that the positive answer would prove the Neggers-Stanley Conjecture for graded naturally labeled posets of width 2 (details in $[R W])$.

Remark 3.1.2. As pointed out by the editor, counterexamples to the Neggers-Stanley Conjecture for the general case $[\mathrm{Br}]$ and for the naturally labeled one [Ste] have been recently announced.

Theorem 3.1.3. The Real Root Conjecture is true if the dimension of the GHS is less than five.

Proposition 3.1.4. Let $h_{X}=\sum_{i=0}^{n} h_{i} t^{i}$ be the h-polynomial of a flag $\mathrm{GHS}^{n-1}$. Then

(1) among the roots of $h_{X}$ with the smallest modulus there is a real negative one, and

(2) if this root is equal to -1 then $X$ is a cross-polytope.

Proof. By the definition of the $h$-polynomial,

$$
\frac{(1+t)^{n}}{h_{X}(-t)}=\left(f_{X}\left(\frac{-t}{1+t}\right)\right)^{-1} \text {. }
$$

In Section 3.3, for the sake of self-consistency of the exposition, we recall a proof of the following well-known proposition:

Proposition 3.1.6. All coefficients in the power series expansion of (3.1.5) are positive.

As a corollary we obtain that there is a pole of (3.1.5) at its convergence radius. Since the modulus of any pole is not smaller than the convergence radius, among the poles of (3.1.5) with the smallest modulus there is a real one. As the poles of (3.1.5) have opposite values to the zeros of $h_{X}$, this proves (1). 
If the root with the smallest modulus is -1 , then, by reciprocity, it has the greatest modulus, and it follows that all roots lie on the unit circle. Thus the linear term, which is the negative of the sum of these roots, is less than or equal to the degree. However, Lemma 2.1.14(1) gives the opposite inequality. The equality is possible only when all roots are equal to -1 . Thus (2) follows from Lemma 2.1.14(2).

Corollary 3.1.7. If $X$ is not a cross-polytope, then $\gamma_{X}$ has a negative real root.

Proof of Theorem 3.1.3. By Remark 3.1.1 we need to check that $\gamma_{X}$ has only real negative roots. If the dimension of the flag GHS $X$ is less than five, then $\gamma_{X}$ is at most quadratic. As $\gamma_{X}$ has real coefficients and, by the previous corollary, at least one real root (if $\gamma_{X}$ is not constant), it cannot have a single nonreal root. Since, by Corollary 2.2.3, all the coefficients of $\gamma_{X}$ are nonnegative, it cannot have a real positive root. Thus the claim is proved.

\subsection{Low-Dimensional Geography}

In this section we prove a partial converse of Theorem 3.1.3.

A quadratic polynomial $1+h_{1} t+t^{2}$ has only real negative roots if and only if $h_{1} \geq 2$. Then it is the $h$-polynomial of an $\left(h_{1}+2\right)$-gon.

A reciprocal polynomial $H$ of odd degree is of the form $H(t)=(1+t) h(t)$ for some reciprocal polynomial $h$. If $H$ has only negative roots then so does $h$. If $h$ is the $h$-polynomial of some complex $X$, then $H$ is the $h$-polynomial of a suspension of $X(X$ joined with $S^{0}$ ).

Therefore a reciprocal monic polynomial with integer coefficients of degree at most three is the $h$-polynomial of a flag sphere triangulation if and only if it has negative real roots. This is almost true if the degree is four (or five, by the previous remark). Namely, we have

Theorem 3.2.1. Let $\gamma$ be a quadratic polynomial with constant term 1 and integer coefficients. Assume that $\gamma(t)-t$ has only negative real roots. Then $h(t)=(1+$ $t)^{4} \gamma\left(t /(1+t)^{2}\right)$ is the h-polynomial of a flag sphere triangulation.

Is the condition that $\gamma(t)-t$ has only negative real zeros essential? In other words, is there a monic reciprocal polynomial of degree four with natural coefficients having only negative real roots such that $h$ is not the $h$-polynomial of a flag sphere triangulation? The smallest example not covered by the above theorem is $1+9 t+21 t^{2}+9 t^{3}+t^{4}$. It is the average of the $h$-polynomials of a join of a 5-gon and an 8-gon and a join of a 6-gon and a 7-gon. However, we state the following conjecture.

Conjecture 3.2.2. Assume that $X$ is a flag triangulation of $S^{3}$ such that $\gamma_{X}(t)-t$ has some nonreal roots. Then $X$ is a join of two polygons. 
For fixed $h_{2}$ there is at most one monic reciprocal polynomial with real negative roots such that $\gamma(t)-t$ has some nonreal roots. It is not an $h$-polynomial of a join of polygons for $h_{2}=21,25,31,35,36,41,43,48,49,54, \ldots$.

Lemma 3.2.3. A polynomial $h(t)=1+h_{1} t+h_{2} t^{2}+h_{1} t^{3}+t^{4}$ has only real negative roots if and only if

$$
\begin{aligned}
\mathbf{c d}:=h_{2}-2 h_{1}+2 & \geq 0, \\
\mathbf{s r}:=h_{1}{ }^{2}-4\left(h_{2}-2\right) & \geq 0, \\
h_{1} & \geq 4 .
\end{aligned}
$$

Proof. First note that if $h(t)=(1+t)^{4}+\gamma_{1} t(1+t)^{2}+\gamma_{2} t^{2}$ then by Remark 3.1.1 we have to show that $\gamma(t)=1+\gamma_{1} t+\gamma_{2} t^{2}$ has only real negative roots. $\gamma$ has only real roots if an only if the discriminant $\mathbf{s r}=\gamma_{1}^{2}-4 \gamma_{2}$ is nonnegative. Then the roots are negative if and only if $\gamma_{2}=\mathbf{c d}$ and $\gamma_{1}=h_{1}-4$ are nonnegative.

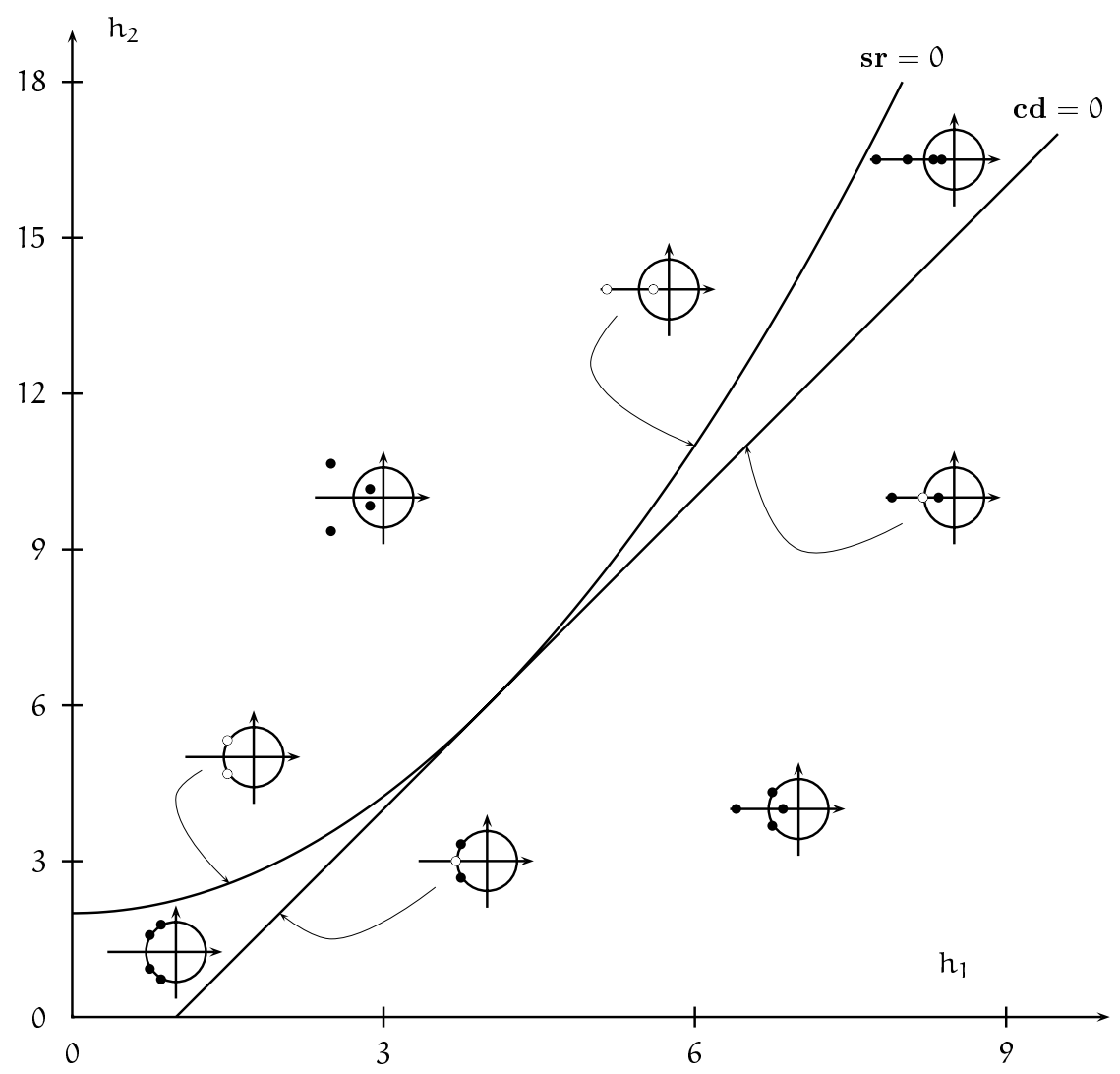

The above figure shows regions in which various configurations of roots appear. White dots mark double roots. The line $\mathbf{c d}=0$ describes the polynomials that have zero at -1 
(of even multiplicity, since $h$ is reciprocal). The other pair of zeros has to be real or lie on the unit circle. In other words, the curve separates the regions with positive and negative values of $h(-1)$ (as in the Charney-Davis Conjecture).

The curve $\mathbf{s r}=0$ describes the polynomials that have a pair of double roots. They have to be real or lie on the unit circle. In other words, the curve is the border of the region where the smallest modulus root is real or all the roots have modulus one.

The above two curves in the $\left(h_{1}, h_{2}\right)$-plane are tangent at the point $(4,6)$. The third inequality separates two domains. In the first all roots are real, while in the second they all lie on the unit circle.

Proof of Theorem 3.2.1. Recall that the $h$-polynomial is multiplicative with respect to the join and the $h$-polynomial of an $m$-gon equals $1+(m-2) t+t^{2}$.

Since $h_{1}=f_{1}-4$ is a natural number, one has

$$
\begin{aligned}
\left\lfloor\frac{h_{1}^{2}}{4}\right\rfloor & =\left\lceil\frac{h_{1}}{2}\right\rceil\left\lfloor\frac{h_{1}}{2}\right\rfloor=\left(h_{1}-\left\lfloor\frac{h_{1}}{2}\right\rfloor\right)\left\lfloor\frac{h_{1}}{2}\right\rfloor \\
& =\max _{k \in \mathbb{N}}\left(h_{1}-k\right) k .
\end{aligned}
$$

Let $\alpha_{k}=h_{2}-2-k\left(h_{1}-k\right)$. In particular, $\mathbf{c d}=\alpha_{2}$. Define

$$
C_{k}=\left\{\left(h_{1}, h_{2}\right) \in \mathbb{Z}^{2}: \alpha_{k-1} \geq 0 \geq \alpha_{k}\right\}
$$

for $k \geq 3$ and $C_{2}=\left\{\left(h_{1}, h_{2}\right) \in \mathbb{Z}^{2}: \alpha_{2}=0, h_{1} \geq 4\right\}$. Then

$$
\left\{\left(h_{1}, h_{2}\right) \in \mathbb{Z}^{2}: 6 \leq 2 h_{1}-2 \leq h_{2} \leq \frac{h_{1}^{2}}{4}+2\right\}=\bigcup_{k=2}^{\infty} C_{k} .
$$

$C_{k}$ is a cone with the vertex at the point $\left(2 k-1, k^{2}-k+2\right)$, which corresponds to the $h$-polynomial of the join of a $(k+1)$-gon and a $(k+2)$-gon and is generated over $\mathbb{N}$ by the primitive vectors $(1, k-1)$ and $(1, k)$.

Assume that $\left(h_{1}-1, h_{2}-2\right)=\left(2 k-1, k^{2}-k+2\right)+a(1, k-1)+b(1, k) \in C_{k}$. Let $X$ be the join of a $(k+1)$-gon and a $(k+2)$-gon subdivided along an edge whose link is a quadrilateral. $X$ has two disjoint edges whose links are a $k$-gon and a $(k+1)$-gon. Subdividing the former $a$ times and the latter $b$ times we obtain a triangulation with the desired $h$-polynomial.

Theorem 3.2.1 should be compared with what is known about the $h$-polynomials of arbitrary (not necessarily flag) sphere triangulations.

Theorem 3.2.4. A reciprocal polynomial $h(t)=\sum_{i=0}^{n} h_{i} t^{i}$ of degree $n=4$ or 5 is the $h$-polynomial of a triangulation of a sphere if and only if

$$
h_{1}\left(h_{1}+1\right) / 2 \geq h_{2} \geq h_{1} \geq h_{0}=1 .
$$

The "if" part is due to Billera and Lee [BL]. The meaning of the inequalities is the 
following:

- $h_{1}\left(h_{1}+1\right) / 2 \geq h_{2}$ is equivalent to the fact that two vertices may be joined by at most one edge.

- $h_{2} \geq h_{1}$ is a part of the Lower Bound Theorem [Ba], [Kal].

- $h_{1} \geq h_{0}$ is equivalent to the fact that a minimum of the number of vertices is achieved on a boundary of a simplex.

\subsection{The Real Root Conjecture for $S^{5}$}

Lemma 3.3.1. If $1+\gamma_{1} t+\gamma_{2} t^{2}+\gamma_{3} t^{3}=(1+x t)(1+y t)(1+z t)$ has only real roots then

$$
\gamma_{2}^{2} \geq 3 \gamma_{3} \gamma_{1}
$$

Proof.

$$
2\left((x y+y z+z x)^{2}-3 x y z(x+y+z)\right)=x^{2}(y-z)^{2}+y^{2}(z-x)^{2}+z^{2}(x-y)^{2} .
$$

Note that if $O$ is the cross-polytope then $\gamma_{O}(t)=1$.

Theorem 3.3.3. Assume that $X$ is a flag triangulation of $S^{5}$ that has an edge $\eta$ whose link is a cross-polytope and $h_{X}(-1)<0$ (the cubical coefficient of $\gamma_{X}$ is positive). Then for a sufficiently large natural number $m$, the $m$-fold subdivision $\operatorname{Sub}_{\eta}^{m}(X)$ of $X$ along $\eta$ contradicts the Real Root Conjecture, i.e. $h_{\mathrm{Sub}_{\eta}^{m}(X)}$ has a nonreal root.

Proof. By (2.4.4), subdivision along $\eta$ increases the linear term of $\gamma_{X}$ without changing other coefficients. However, (3.3.2) for $\gamma_{X}$ is false when $\gamma_{3}=-h_{X}(-1)$ is positive and the linear coefficient $\gamma_{1}$ is sufficiently large.

What is left to do is to exhibit a complex satisfying the hypothesis of Theorem 3.3.3.

Take the join $X_{1}$ of two pentagons. Subdivide an edge whose link is a quadrilateral to obtain $X_{2}$. Finally let $X$ be the join of $X_{2}$ and another pentagon. $X_{1}$ has a vertex (the new one) whose link is a cross polytope. Thus, $X$ has an edge whose link is a cross polytope (join of the above with any edge of the pentagon).

By (2.4.4) and (2.4.5) we calculate

$$
\gamma_{X}(t)=(1+t)\left((1+t)^{2}+t \cdot 1\right)=1+4 t+4 t^{2}+t^{3} .
$$

Therefore $X$ satisfies the hypothesis of Theorem 3.3.3 and, twice subdivided, $X$ becomes a counterexample to the Real Root Conjecture. In fact, it suffices to take a single subdivision, but this requires an extra check. This (smallest known) counterexample to the Real Root Conjecture has $f$-polynomial $f_{\mathrm{Sub}_{\eta} X}(t)=1+17 t+109 t^{2}+345 t^{3}+575 t^{4}+$ $483 t^{5}+161 t^{6}$.

The above example still satisfies Conjecture 2.1.7 and, in particular, the CharneyDavis Conjecture (see Corollary 2.4.7). 
Corollary 3.3.4. Taking the join with any flag sphere triangulations one finds that there are counterexamples to the Real Root Conjecture if the dimension of the sphere is greater than or equal to five.

Other counterexamples to the Real Root Conjecture are presented in a forthcoming paper [Ga].

\subsection{The Smallest Root}

We briefly present the classical proof of Proposition 3.1.6 that uses Coxeter groups. Another homological proof can be given by showing that the Stanley-Reisner face ring $R(X)$ of a flag complex $X$ is Koszul, thus the coefficient of (3.1.5) at $t^{j}$ equals $\operatorname{dimTor}_{j}^{R(X)}(k, k)$ (see, e.g. Proposition 4.13 of [RW]).

With any flag complex $X$ with a vertex set $S$ one associates a right-angled Coxeter group $W$ with the following presentation:

$$
\left.W=\langle S| s^{2}=1 \text { for all } s \in S, s t=t s \text { for all }\{s, t\} \in X\right\rangle .
$$

The definition of $W$ uses only the one-skeleton of $X$, but the following observation links the whole $X$ to $W$. Let the subgroup of $W$ generated by $T \subset S$ be denoted by $W_{T}$. Then $W_{T}$ is finite if and only if $T \in X$. In this case $W_{T}=(\mathbb{Z} / 2)^{\# T}$.

Definition 3.4.1. Define a formal series $W(t)=\sum_{w \in W} t^{\ell(w)}$, where $\ell$ denotes the length function with respect to the generating set $S$. We call $W(\cdot)$ the growth series of $W$.

Proposition 3.4.2 [Se]. $\quad W(t)$ represents a rational function. Moreover, if $W$ is infinite, then

$$
\frac{1}{W(t)}=\sum_{T \subset S} \frac{(-1)^{\# T}}{W_{T}\left(t^{-1}\right)},
$$

where $T$ runs over subsets of $S$ such that $W_{T}$ is finite.

Corollary 3.4.3. Let $W$ be the Coxeter group associated to a flag complex X. Then

$$
f_{X}\left(\frac{-t}{1+t}\right)=\frac{1}{W(t)} .
$$

Proof. If $W_{T}$ is finite, then $W_{T}(t)=(1+t)^{\# T}$, thus Proposition 3.4.2 reduces to (3.4.4).

This finishes the proof of Proposition 3.1.6.

Note that in proving Proposition 3.1.4(1) we did not use the assumption that $X$ is a GHS. The second part of Proposition 3.1.4 allows the following generalization (one may consult Proposition 3.10 of [DDJO] for ample discussion): 
Proposition 3.4.5. If $X$ is any flag complex and the radius of convergence of $W_{X}(\cdot)$ equals one (i.e. $h_{X}$ has no zeros in the interior of the unit disk), then $X$ is a join of a cross-polytope and a simplex (i.e. a multiple suspension of a simplex).

Proof. Let $T$ be any subset of $S$. The coefficients of $W_{T}(\cdot)$ are dominated by those of $W(\cdot)$. This is straightforward either by noticing that the length function on $W_{T}$ is the restriction of the length function on $W$ or by interpreting the coefficients as dimensions of Tor modules.

Thus the convergence radius of $W_{T}(\cdot)$ is greater than or equal to that of $W(\cdot)$.

Let $x$ and $y$ be two vertices not joined by an edge (if there is no such pair then $X$ is a simplex and we are done). We want to show that $X$ is a suspension of its subcomplex spanned by $S-\{x, y\}$. To do this we need to show that if $z$ is any vertex different from $x$ and $y$ then $z$ is joined with both $x$ and $y$. If not, then straightforward computation, using Proposition 3.4.2, shows that the convergence radius of $W_{\{x, y, z\}}(\cdot)$ is strictly smaller than one.

\section{Acknowledgements}

The author thanks Tadeusz Januszkiewicz and Vic Reiner for useful discussions and Andrzej Derdziński and Pawel Goldstein for their extensive help with the final version of this manuscript.

\section{References}

[Ba] D. Barnette, A proof of the lower bound conjecture for convex polytopes, Pacific J. Math. 46 (1973), 349-354.

[Br] P. Brändén, Counterexamples to the Neggers-Stanley Conjecture, Electron. Res. Announc. Amer. Math. Soc. 10 (2004), 155-158.

[BL] L. Billera and C. Lee, Sufficiency of McMullen's conditions for $f$-vectors of simplicial polytopes, Bull. Amer. Math. Soc. 2 (1980), 181-185.

[C] J. W. Cannon, Shrinking cell-like decompositions of manifolds. Codimension three, Ann. Math. 110 (1979), 83-112.

[CD] R. Charney and M. Davis, The Euler characteristic of a nonpositively curved, piecewise Euclidean manifold, Pacific J. Math. 171 (1995), 117-137.

[DDJO] M. Davis, J. Dymara, T. Januszkiewicz and B. Okun, Weighted $L^{2}$-cohomology of Coxeter groups, arXiv:math.GT/0402377.

[DO] M. Davis and B. Okun, Vanishing theorems and conjectures for the $L^{2}$-homology of right-angled Coxeter Groups, Geom. Topol. 5 (2001), 7-74.

[E] R. D. Edwards, The topology of manifolds and cell-like maps, Proc. ICM Helsinki, 1978, pp. 111-127.

[F] M. H. Freedman, The topology of four-dimensional manifolds, J. Differential Geom. 17(3) (1982), $357-453$.

[Ga] S. R. Gal, On normal subgroups of coxeter groups generated by standard parabolic subgroups, to appear in Geom. Dedicata.

[Gr] M. Gromov, Hyperbolic Groups, in Essays in Group Theory, S. G. Gersten, ed., pp. 75-263, MSRI Publ. 8, Springer-Verlag, Berlin, 1987.

[Kal] G. Kalai, Rigidity and the lower bound theorem, I., Invent. Math. 88(1) (1987), 125-151.

[Kar] K. Karu, The cd-index of fans and lattices, arXiv:math.AG/0410513.

[Kl] V. Klee, A combinatorial proof of Poincaré's duality theorem, Canad. J. Math. 16 (1964), 517-531. 
[RW] V. Reiner and V. Welker, On the Charney-Davis and Neggers-Stanley Conjectures, J. Combin. Theory Ser. A 109(2) (2005), 247-280.

[Se] J. P. Serre, Cohomologie des groupes discrets, in Prospects in Mathematics, pp. 77-169, Annal of Mathematical Studies No. 70, Princeton University Press, Princeton, NJ, 1971.

[Sta1] R. Stanley, Combinatorics and Commutative Algebra, Birkhäuser, Boston, MA, 1996.

[Sta2] R. Stanley, Flag $f$-vectors and the cd-index, Math. Z. 216 (1994), 483-499.

[Ste] J. Stembridge, Counterexamples to the poset conjecture of Neggers, Stanley, and Stembridge, to appear in Trans. Amer. Math. Soc.

Received April 12, 2004, and in revised form October 11, 2004, and January 4, 2005.

Online publication May 27, 2005. 